\title{
Sobolev and Morrey Estimates for Non-Smooth Vector Fields of Step Two
}

\author{
A. Montanari and D. Morbidelli
}

\begin{abstract}
We prove Sobolev-type and Morrey-type inequalities for Sobolev spaces related to a family of non-smooth vector fields which formally satisfy the Hörmander condition of step 2. The coefficients of the vector fields are not regular enough to define the Carnot-Carathéodory distance. Thus the result is proved by developing a real analysis technique which is based on an approximation procedure of Lipschitz continuous vector fields with a family of left-invariant first order operators on a nilpotent Lie group.
\end{abstract}

Keywords: Sobolev inequalities, Freezing method, homogeneous spaces

AMS subject classification: $46 \mathrm{E} 35$

\section{Introduction}

In this paper we are concerned with the Sobolev- and Morrey-type inequalities

$$
\begin{aligned}
\left(\int_{\Omega}|f(x)|^{\frac{p Q}{Q-p}} d x\right)^{\frac{Q-p}{p Q}} & \leq c_{1}\left(\int_{\Omega}|X f(x)|^{p} d x\right)^{\frac{1}{p}} \quad(1 \leq p<Q) \\
|f(y)-f(z)| & \leq c_{2}\left(\int_{\Omega}|X f(x)|^{p} d x\right)^{\frac{1}{p}} \rho(y, z)^{\alpha} \quad(y, z \in \Omega, p>Q \Omega 2)
\end{aligned}
$$

Here $\Omega \subset \mathbb{R}^{n}$ is a bounded set, $f \in C_{0}^{1}(\Omega)$ and $X f=\left(X_{1} f, \ldots, X_{m} f\right)$ is the degenerate gradient associated with a family of vector fields of the form

$$
X_{j}=\frac{\partial}{\partial x_{j}}+\sum_{k=m+1}^{n} a_{j}^{k}(x) \frac{\partial}{\partial x_{k}} \quad(j=1, \ldots, m ; m \leq n),
$$

$Q=m+2(n-m)$ denotes the "homogeneous dimension" associated with the $X_{j}, \alpha<1$ is a suitable positive number and $\rho$ is a quasi-distance naturally associated with the vector fields.

Both authors: Univ. di Bologna, Dip. di Mat., P. di Porta S. Donato 5, 40126 Bologna, Italy; montanar@dm.unibo.it and morbidel@dm.unibo.it

ISSN 0232-2064 / \$2.50 (C) Heldermann Verlag Berlin 
Historically, the first embedding properties for degenerate Sobolev spaces are essentially contained in the papers $[6,7,10,18]$ where the "subelliptic estimate"

$$
\|f\|_{H^{\varepsilon}} \leq c\left(\|f\|_{L^{2}}+\|X f\|_{L^{2}}\right) \quad\left(f \in C_{0}^{\infty}(\Omega)\right)
$$

is proved. Here $H^{\varepsilon}$ denotes the classical fractional Sobolev space of a suitable order $\varepsilon \in(0,1]$. Obviously, the classical embedding $H^{\varepsilon} \subset L^{\frac{2 n}{n-2 \varepsilon}}$ furnishes the estimate

$$
\|f\|_{L^{\frac{2 n}{n-2 \varepsilon}}} \leq c\left(\|f\|_{L^{2}}+\|X f\|_{L^{2}}\right) \quad\left(f \in C_{0}^{\infty}(\Omega)\right) .
$$

A different class of significant integral inequalities involving vector fields (see, for example, $[10,19]$ ) are the Poincaré-type inequalities

$$
\int_{B}\left|f-f_{B}\right| \leq c_{1} r \int_{c_{2} B}|X f| \quad\left(f \in C^{1}\left(c_{2} B\right)\right)
$$

where $B$ is a ball of radius $r$ with respect to the Carnot-Carathéodory distance (see [6] for the definition), $c_{2} B$ is the ball with same center of $B$ and radius $c_{2} r$ and $f_{B}=|B|^{-1} \int_{B} f$ denotes the average of $f$ on the ball. In recent years, many papers have been devoted to the study of the so-called self-improving properties of Poincaré inequalities (see, e.g., $[1,11,13,17,22]$ ). In these papers it is proved that the Poincaré inequality, together with the doubling property of the Lebesgue measure of the Carnot-Carathéodory balls, are sufficient to construct a satisfactory theory of first order Sobolev spaces.

While this last "axiomatic" aspect of the theory has been developed in great generality, it seems that the direct proof of inequalities (1) - (3) or (5) in concrete situations is a very complicate matter. There are essentially two classes of examples of vector fields in $\mathbb{R}^{n}$ for which the proof of the mentioned inequalities is available: the vector fields satisfying Hörmander condition (see $[18,19])$ and those of Grushin-Franchi-Lanconelli type (see $[8,10])$. In the first case smooth regularity is required, while in the latter the vector fields have to be of "diagonal form".

In the present paper we study embedding properties for Sobolev paces associated with families of step two which are in general non-smooth and non-diagonal. Before stating our results we briefly quote some recent papers in which this problem is addressed.

A first embedding result for non-smooth and non-diagonal vector fields of step two is contained in [20], where (3) is proved with $\varepsilon=\frac{1}{2}$ by means of a Fourier analysis method. This procedure only requires that the coefficients of the vector fields have second derivatives in a suitable $L^{p}$-space. However, since the Sobolev-type inequality is obtained through (4), the embedding exponent is in general not sharp. 
For a couple of $C^{1}$ vector fields of step two in $\mathbb{R}^{3}$, inequalities (1) and (2) are consequences of the Poincaré inequality (5), which has been proved in [21]. The technique of the paper [21] relies on precise estimates of the CarnotCarathéodory distance. In the regularity assumptions of our paper it is not even clear if the mentioned distance is finite for each pair of points.

In the recent paper [4] the Sobolev inequality (1) is proved for a pair of Lipschitz continuous vector fields in $\mathbb{R}^{3}$, which arise in the study of regularity properties of real hypersurfaces in $\mathbb{C}^{2}$ with Levi curvature belonging to a suitable Sobolev space and different from zero at every point. This result shows that embedding theorems for Sobolev spaces related to vector fields with non-smooth coefficients are an important tool in the study of regularity properties of some nonlinear elliptic degenerate equations. The paper [4] has motivated the present one.

Our main result is the following.

Theorem 1.1. Consider the vector fields in $\mathbb{R}^{n}$

$$
X_{j}=\partial_{j}+\sum_{k=m+1}^{n} a_{j}^{k} \partial_{k}
$$

with $a_{j}^{k} \in \operatorname{Lip}\left(\mathbb{R}^{n}\right)$. Let $Q=m+2(n-m)$. Assume that, for almost every $x \in \mathbb{R}^{n}$,

$$
\partial_{j}=\sum_{1 \leq i<k \leq m} \lambda_{j}^{i, k}(x)\left[X_{i}, X_{k}\right](x) \quad(j=m+1, \ldots, n)
$$

where

$$
\lambda_{j}^{i, k}, X_{h} \lambda_{j}^{i, k} \in L^{\infty} \quad(j=m+1, \ldots, n ; i, k, h=1, \ldots, m) .
$$

(here $X_{h}$ and $\left[X_{i}, X_{k}\right]=X_{i} X_{k}-X_{k} X_{i}$ denote distributional derivatives). Then, for any bounded open set $\Omega \subset \mathbb{R}^{n}$, the following statements hold:

(A) (Sobolev inequality). If $1 \leq p<Q$, then

$$
\|f\|_{L^{\frac{p Q}{Q-p}}} \leq c_{1}\|X f\|_{L^{p}}
$$

for all $f \in C_{0}^{1}(\Omega)$.

(B) (Trudinger inequality). If $p=Q$, then

$$
\int_{\Omega} \exp \left(\left(\frac{|f(x)|}{c_{2}\|X f\|_{Q}}\right)^{\frac{Q}{Q-1}}\right) d x \leq c_{3}
$$


for all $f \in C_{0}^{1}(\Omega)$.

(C) (Morrey-type estimate). There exists a quasi-distance $\rho$ in $\Omega$ satisfying

$$
k_{1}|x-y| \leq \rho(x, y) \leq k_{2}|x-y|^{\frac{1}{2}}
$$

for all $x, y \in \Omega\left(\right.$ with $|\cdot|$ the Euclidean norm in $\left.\mathbb{R}^{n}\right)$ and such that, if $Q<$ $p<\infty$, then

$$
|f(x)-f(z)| \leq c_{4}\|X f\|_{L^{p}} \rho(x, z)^{\alpha} \quad(x, z \in \Omega)
$$

for all $f \in C_{0}^{1}(\Omega)$ where $\alpha=\min \left\{\frac{1}{2}, 1-\frac{Q}{p}\right\}$.

(D) If $1<p<Q$, then the space $W_{0}^{1, p}$ is compactly embedded into $L^{q}$ for any $q<\frac{Q p}{Q-p}$ where $W_{0}^{1, p}(\Omega)$ denotes the closure of $C_{0}^{\infty}(\Omega)$ with respect to the norm $\|X f\|_{L^{p}(\Omega)}$.

The constants $k_{1}$ and $k_{2}$ depend on $\Omega$ and on the Lipschitz constants of the $a_{j}^{k}$ 's in $(6) w$. The constants $c_{1}, c_{2}, c_{3}, c_{4}$ depend on $p, \Omega$, on the Lipschitz

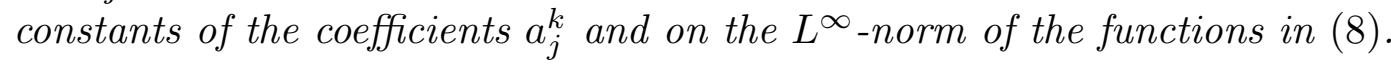

Hypotheses (6) and (7) in the smooth case ensure Hörmander condition of step 2. Let us remark that hypothesis (6) is not restrictive. Indeed, given a family $X_{1}, \ldots, X_{m}$ of Lipschitz continuous vector fields linearly independent at a point $\bar{x}$, we can find a new family $Y_{j}=\sum_{j=1}^{m} M_{j, k} X_{k} \quad(j=1, \ldots, m)$ of form (6), where $\left(M_{j, k}\right)_{j, k=1, \ldots, m}$ is a non-singular matrix with Lipschitz continuous entries in a neighborhood of $\bar{x}$.

Our method is inspired to some results of Rothschild and Stein [23] and to those of Citti, Lanconelli and Montanari [4], where a version of (1) for a pair of vector fields in $\mathbb{R}^{3}$ is proved. The main idea is to approximate the operators $X_{j}$ at each fixed point with a family of vector fields which are left invariant on a nilpotent Lie group of step two. However, Rothschild and Stein in [23] first introduce local coordinates naturally associated with the vector fields and then, in this new system of canonical coordinates, they approximate the vector fields. Their procedure requires high regularity on the coefficients. Since our coefficients are only Lipschitz continuous, we will first approximate the vector fields with a family of smooth and left invariant first order operators on a nilpotent Lie group of step two and then we will introduce a change of variables. Our "freezing method" has been first introduced by Citti in [2] for $C^{1, \alpha}$ coefficients and then adapted to Lipschitz continuous vector fields in [3]. A consequence of our different approximation appears for $p>2 Q$, since the exponent $\alpha=\min \left(\frac{1}{2}, 1-\frac{Q}{p}\right)$ in Morrey estimate, for a function $f$ with degenerate gradient in $L^{p}$, is worse than the usual exponent $1-\frac{Q}{p}$, which arises in the smooth case. 
We conclude the introduction by remarking that our results hold in the class of compactly supported functions. This is a consequence of the fact that our method relies on several integrations by part. Moreover, the quasidistance $\rho$ in Morrey estimate is not the Carnot-Carathéodory distance. It would be interesting to study the Carnot-Carathéodory distance and to discuss the validity of a Poincaré inequality (for non-compactly supported functions) in the regularity assumptions of the present paper.

The present paper is organized as follows. In Section 2 we introduce at any point $\bar{x} \in \mathbb{R}^{n}$ some "frozen" vector fields $X_{j, \bar{x}}$ and we show that there exists an explicit change of variable which transforms them into a nilpotent family independent on the point $\bar{x}$. We then define the quasi-distance $\rho$ and get various estimates of derivatives of the fundamental solution $\Gamma_{\bar{x}}$ of the operator $\sum_{j=1}^{m} X_{j, \bar{x}}^{2}$ in term of $\rho$. In Section 3 we prove statements $(\mathrm{A})$ and (B) of Theorem 1.1 by means of a representation formula for $f \in C_{0}^{1}(\Omega)$ in term of $\Gamma_{\bar{x}}$. In Section 4 we prove statements (C) and (D) of Theorem 1.1 by varying the point $\bar{x}$ in our representation formula.

In this paper we will use the following notations:

$c$ is a generic constant which may change even in a single string of estimates; we write $a \simeq b$ to state that there exist two constants $c_{1}>0$ and $c_{2}>0$ such that $c_{1} b \leq a \leq c_{2} b ;\|f\|_{p}$ or $\|f\|_{L^{p}}$ will denote the $L^{p}$-norm of a function $f$ on the set $\Omega ;|A|$ stands for the Lebesgue measure of the set $A \subset \mathbb{R}^{n}$; a vector field $X=\sum_{k=1}^{n} a_{k}(x) \partial_{k}$ will be identified with the vector function $\left(a_{1}(x), \ldots, a_{n}(x)\right)$.

\section{Freezing method and the quasi-distance $\rho$}

In this section, starting from a family of $m$ vector fields of form (9), we introduce at any point $\bar{x} \in \mathbb{R}^{n}$ some "frozen" vector fields $X_{j, \bar{x}}$ and we show that the latter (modulo an explicit change of variable) can be transformed into a family $H_{j}(j=1, \ldots, m)$ which does not depend on the point $\bar{x}$.

Consider in $\mathbb{R}^{n}$ the vector fields

$$
X_{j}=\partial_{j}+\sum_{k=m+1}^{n} a_{j}^{k} \partial_{k} \quad(j=1, \ldots, m)
$$

where the $a_{j}^{k}=a_{j}^{k}(x)$ are Lipschitz continuous real-valued functions defined on $\mathbb{R}^{n}$. Assume that the fields are of step 2, in the sense of (7). This ensures that the total number $\frac{m(m-1)}{2}$ of commutators $\left[X_{i}, X_{j}\right] \quad(1 \leq i<j)$ satisfies $\frac{m(m-1)}{2} \geq n-m$. Fix a family

$$
\mathcal{A} \subset\{(i, l): 1 \leq i<l \leq m\}
$$


with $n-m$ objects and a bijective correspondence

$$
\begin{aligned}
& g:\{m+1, \ldots, n\} \rightarrow \mathcal{A}, \quad g(k)=\left(g_{1}(k), g_{2}(k)\right) \\
& h=g^{-1}: \mathcal{A} \rightarrow\{m+1, \ldots, n\} .
\end{aligned}
$$

For any $k=m+1, \ldots, n$ and $i, j=1, \ldots, m$ let

$$
b_{i, j}^{k}= \begin{cases}1 & \text { if }(i, j)=g(k) \\ 0 & \text { otherwise }\end{cases}
$$

Define for any fixed $\bar{x} \in \mathbb{R}^{n}$

$$
\begin{aligned}
a_{j, \bar{x}}^{k}(x) & =a_{j}^{k}(\bar{x})+\sum_{i=1}^{m} b_{i, j}^{k}\left(x_{i}-\bar{x}_{i}\right) \quad\left(x \in \mathbb{R}^{n}\right) \\
X_{j, \bar{x}} & =\partial_{j}+\sum_{k=m+1}^{n} a_{j, \bar{x}}^{k} \partial_{k} .
\end{aligned}
$$

In the sequel we will use the following elementary properties:

$$
\begin{aligned}
& \left|a_{j, \bar{x}}^{k}(x)-a_{j}^{k}(x)\right| \leq c|x-\bar{x}| \\
& X_{i} a_{j, \bar{x}}^{k}(x)-X_{i} a_{j, \bar{y}}^{k}(x)=0
\end{aligned}
$$

where the constant $c$ depends on the Lipschitz constants of the $a_{j}^{k}$ 's.

If $1 \leq l<j \leq m$, then

$$
\begin{aligned}
{\left[X_{l, \bar{x}}, X_{j, \bar{x}}\right]=\partial_{l}\left(\sum_{\substack{k=m+1, \ldots, n \\
i=1, \ldots, m}} b_{i, j}^{k}\left(x_{i}-\bar{x}_{i}\right) \partial_{k}\right) } \\
\quad-\partial_{j}\left(\sum_{\substack{k=m+1, \ldots, n \\
i=1, \ldots, m}} b_{i, l}^{k}\left(x_{i}-\bar{x}_{i}\right) \partial_{k}\right) \\
=\sum_{k>m}\left(b_{l, j}^{k} \partial_{k}-b_{j, l}^{k} \partial_{k}\right) \\
=\sum_{k>m} b_{l, j}^{k} \partial_{k} \\
=\frac{\partial}{\partial x_{h(l, j)}} .
\end{aligned}
$$


Define, for a fixed $\bar{x} \in \mathbb{R}^{n}$ and for all $u \in \mathbb{R}^{n}$,

$$
\begin{aligned}
\phi_{\bar{x}}(u) & =\exp \left(\sum_{j=1}^{m} u_{j} X_{j, \bar{x}}+\sum_{(l, j) \in \mathcal{A}} u_{h(l, j)}\left[X_{l, \bar{x}}, X_{j, \bar{x}}\right]\right)(\bar{x}) \\
& =\exp \left(\sum_{j=1}^{m} u_{j} X_{j, \bar{x}}+\sum_{(l, j) \in \mathcal{A}} u_{h(l, j)} \partial_{h(l, j)}\right)(\bar{x}) \\
& =\exp \left(\sum_{j=1}^{m} u_{j} X_{j, \bar{x}}+\sum_{k=m+1}^{n} u_{k} \partial_{k}\right)(\bar{x}) .
\end{aligned}
$$

The exponential map $\phi_{\bar{x}}(u)$ can be explicitly written by solving the Cauchy problem

$$
\begin{aligned}
& \dot{\gamma}(s)=\sum_{j=1}^{m} u_{j}\left\{e_{j}+\sum_{k=m+1}^{n}\left(a_{j}^{k}(\bar{x})+\sum_{i=1}^{j-1} b_{i, j}^{k}\left(\gamma_{i}(s)-\bar{x}_{i}\right)\right) e_{k}\right\}+\sum_{k=m+1}^{n} u_{k} e_{k} \\
& \gamma(0)=\bar{x}
\end{aligned}
$$

and letting $\phi_{\bar{x}}(u)=\gamma(1) \quad\left(e_{j}\right.$ denotes the Euclidean versor $)$. If

$$
\begin{array}{ll}
i \leq m, & \text { then } \gamma_{i}(s)=\bar{x}_{i}+u_{i} s \\
k>m, & \text { then } \dot{\gamma}_{k}(s)=\sum_{j=1}^{m} u_{j} a_{j}^{k}(\bar{x})+\sum_{1 \leq i<j \leq m} u_{j} b_{i, j}^{k} u_{i} s+u_{k} .
\end{array}
$$

Thus, integrating over $[0,1]$,

$$
\phi_{\bar{x}}(u)=\gamma(1)=\sum_{j=1}^{m}\left(\bar{x}_{j}+u_{j}\right) e_{j}+\sum_{j=m+1}^{n}\left(\bar{x}_{j}+u_{j}+A^{j}(\bar{x}) \cdot u+\frac{1}{2} B^{j} u \cdot u\right) e_{j}
$$

where we have introduced the notation

$$
\begin{aligned}
A^{j}(\bar{x}) \cdot u & =\sum_{i=1}^{m} a_{i}^{j}(\bar{x}) u_{i} \\
B^{j} u \cdot v & =\sum_{i, l=1}^{m} b_{i, l}^{j} u_{i} v_{l}=u_{g_{1}(j)} v_{g_{2}(j)}
\end{aligned}
$$

(the last equality follows from (11)). Denote by $\psi_{\bar{x}}$ the inverse map of $\phi_{\bar{x}}$. Then

$$
\begin{aligned}
u=\psi_{\bar{x}}(x)= & \sum_{j=1}^{m}\left(x_{j}-\bar{x}_{j}\right) e_{j} \\
& +\sum_{j=m+1}^{n}\left\{x_{j}-\bar{x}_{j}-(x-\bar{x}) \cdot A^{j}(\bar{x})-\frac{1}{2} B^{j}(x-\bar{x}) \cdot(x-\bar{x})\right\} e_{j} .
\end{aligned}
$$


In order to write now the vector field $X_{j, \bar{x}}$ in the coordinates $u$ recall first that, if $j=m+1, \ldots, n$, then $\frac{\partial}{\partial x_{j}}=\frac{\partial}{\partial u_{j}}$. If $j \leq m$, the derivative $\partial_{j}$ transforms as follows:

$$
\begin{aligned}
\frac{\partial}{\partial x_{j}} & =\sum_{k=1}^{n} \frac{\partial u_{k}}{\partial x_{j}} \frac{\partial}{\partial u_{k}} \\
& =\sum_{k=1}^{m} \frac{\partial u_{k}}{\partial x_{j}} \frac{\partial}{\partial u_{k}}+\sum_{k=m+1}^{n} \frac{\partial u_{k}}{\partial x_{j}} \frac{\partial}{\partial u_{k}} \\
& =\frac{\partial}{\partial u_{j}}+\sum_{k=m+1}^{n} \frac{\partial}{\partial x_{j}}\left\{(x-\bar{x})_{k}-A^{k}(\bar{x}) \cdot(x-\bar{x})-\frac{1}{2} B^{k}(x-\bar{x}) \cdot(x-\bar{x})\right\} \frac{\partial}{\partial u_{k}} \\
& =\frac{\partial}{\partial u_{j}}+\sum_{k=m+1}^{n}\left\{-a_{j}^{k}(\bar{x})-\frac{\partial}{\partial x_{j}}\left(\frac{1}{2} B^{k}(x-\bar{x}) \cdot(x-\bar{x})\right)\right\} \frac{\partial}{\partial u_{k}} \\
& =\frac{\partial}{\partial u_{j}}-\sum_{k=m+1}^{n} a_{j}^{k}(\bar{x}) \frac{\partial}{\partial x_{k}}-\frac{1}{2} \sum_{k=m+1}^{n} \frac{\partial}{\partial x_{j}}\left((x-\bar{x})_{g_{1}(k)} \cdot(x-\bar{x})_{g_{2}(k)}\right) \frac{\partial}{\partial x_{k}}
\end{aligned}
$$

where we used the property $B^{k} \xi \cdot \xi=\xi_{g_{1}(k)} \xi_{g_{2}(k)}$. Then the definition of $X_{j, \bar{x}}$ gives

$$
\begin{aligned}
X_{j, \bar{x}}= & \frac{\partial}{\partial x_{j}}+\sum_{k=m+1}^{n} a_{j}^{k}(\bar{x}) \frac{\partial}{\partial x_{k}}+\sum_{\substack{k=m+1, \ldots, n \\
i=1, \ldots, m}} b_{i, j}^{k}\left(x_{i}-\bar{x}_{i}\right) \frac{\partial}{\partial x_{k}} \\
= & \frac{\partial}{\partial u_{j}}-\frac{1}{2} \sum_{k=m+1}^{n} \frac{\partial}{\partial x_{j}}(x-\bar{x})_{g_{1}(k)}(x-\bar{x})_{g_{2}(k)} \frac{\partial}{\partial x_{k}}+\sum_{\substack{k=m+1, \ldots, n \\
i=1, \ldots, m}} b_{i, j}^{k}(x-\bar{x})_{i} \frac{\partial}{\partial x_{k}} \\
= & \frac{\partial}{\partial u_{j}}-\frac{1}{2} \sum_{\left\{k: g_{1}(k)=j\right\}}(x-\bar{x})_{g_{2}(k)} \frac{\partial}{\partial x_{k}} \sum_{\left\{k: g_{2}(k)=j\right\}}(x-\bar{x})_{g_{1}(k)} \frac{\partial}{\partial x_{k}}+\sum_{k=m+1}^{n} \sum_{\{i:(i, j)=g(k)\}}(x-\bar{x})_{i} \frac{\partial}{\partial x_{k}} \\
= & \frac{\partial}{\partial u_{j}}-\frac{1}{2} \sum_{\left\{k: g_{1}(k)=j\right\}} u_{g_{2}(k)} \frac{\partial}{\partial u_{k}}+\frac{1}{2} \sum_{\left\{k: g_{2}(k)=j\right\}} u_{g_{1}(k)} \frac{\partial}{\partial u_{k}} \\
= & \frac{\partial}{\partial u_{j}}-\frac{1}{2} \sum_{\{l:(j, l) \in \mathcal{A}\}} u_{l} \frac{\partial}{\partial u_{h(j, l)}}+\frac{1}{2} \sum_{\{l:(l, j) \in \mathcal{A}\}} u_{l} \frac{\partial}{\partial u_{h(l, j)}} \\
= & H_{j} .
\end{aligned}
$$

The vector fields $H_{j}(j=1, \ldots, m)$ are homogeneous of degree 1 with respect to the family of dilations $(D(\lambda))_{\lambda>0}$ defined by

$$
D(\lambda)\left(\xi_{1}, \ldots, \xi_{n}\right)=\left(\lambda \xi_{1}, \ldots, \lambda \xi_{m}, \lambda^{2} \xi_{m+1}, \ldots, \lambda^{2} \xi_{n}\right) \quad\left(\xi \in \mathbb{R}^{n}\right) .
$$


Moreover, the vector fields $H_{j}$ are left invariant on the Lie Group $\left(\mathbb{R}^{n}, \circ\right)$, where the law "o" is defined for $u, v \in \mathbb{R}^{n}$ by

$$
u \circ v=\sum_{j=1}^{m}\left(u_{j}+v_{j}\right) e_{j}+\sum_{j=m+1}^{n}\left(u_{j}+v_{j}+\frac{1}{2}\left(B^{j} u \cdot v-B^{j} v \cdot u\right)\right) e_{j} .
$$

Obviously, $u^{-1}=-u$ and

$$
u^{-1} \circ v=\sum_{j=1}^{m}\left(v_{j}-u_{j}\right) e_{j}+\sum_{j=m+1}^{n}\left(v_{j}-u_{j}-\frac{1}{2}\left(B^{j} u \cdot v-B^{j} v \cdot u\right)\right) e_{j} \text {. }
$$

We now use the change of variable $\phi_{\bar{x}}$ in order to get precise estimates of the fundamental solution (and of its derivatives) of the operator $L_{\bar{x}}=$ $\sum_{j=1}^{m} X_{j, \bar{x}}^{2}$ in term of a suitable quasi-distance.

Proposition 2.1. Let $\Omega \subset \mathbb{R}^{n}$ be a bounded set. Let $a_{j}^{k} \in \operatorname{Lip}(\Omega) \quad(j=$ $1, \ldots, m ; k=m+1, \ldots, n)$. Consider the function

$$
\rho(x, y)=\sum_{j=1}^{m}\left|y_{j}-x_{j}\right|+\sum_{j=m+1}^{n}\left|(y-x)_{j}-A^{j}(x) \cdot(y-x)\right|^{\frac{1}{2}} \quad(x, y \in \Omega) .
$$

Then there exist two constants $c_{1}>0$ and $c_{2}>0$ depending on $\Omega$ and on the Lipschitz norm of $a_{j}^{k}$, such that

$$
\left.\begin{array}{l}
\rho(x, y) \leq c_{1} \rho(y, x) \\
\rho(x, y) \leq c_{2}(\rho(x, z)+\rho(z, y))
\end{array}\right\} \quad(x, y, z \in \Omega)
$$

Moreover, there are constants $c_{3}>0$ and $c_{4}>0$ such that

$$
\begin{gathered}
c_{3}^{-1}|x-y| \leq \rho(x, y) \leq c_{3}|x-y|^{\frac{1}{2}} \quad(x, y \in \Omega) \\
|\{y: \rho(x, y)<r\}|=c_{4} r^{Q} \quad(r>0, Q=m+2(n-m)) .
\end{gathered}
$$

Proof. We start by showing (19) and estimate

$$
\begin{aligned}
|x-y| & \leq \sum_{j=1}^{m}\left|x_{j}-y_{j}\right|+\sum_{j=m+1}^{n}\left\{\left|x_{j}-y_{j}-A^{j}(x) \cdot(y-x)\right|+\left|A^{j}(x) \cdot(y-x)\right|\right\} \\
& \leq \sum_{j=1}^{m}\left|x_{j}-y_{j}\right|+c \sum_{j=m+1}^{n}\left|x_{j}-y_{j}-A^{j}(x) \cdot(y-x)\right|^{\frac{1}{2}}+c \sum_{j=1}^{m}\left|y_{j}-x_{j}\right| \\
& \leq c \rho(x, y)
\end{aligned}
$$


Here we have used the boundedness of $\Omega$ and of $\left|A^{j}(x)\right| \quad(x \in \Omega)$. Again, by the boundedness of $\Omega$ and of $\left|A^{j}(x)\right| \quad(x \in \Omega)$ together with the Euclidean triangle inequality, we get the estimate $\rho(x, y) \leq c|x-y|^{\frac{1}{2}}$.

In order to check that $\rho$ is a quasi-distance we first prove that $\rho(x, y) \leq$ $c \rho(y, x)$. Indeed,

$$
\begin{aligned}
\rho(x, y)= & \sum_{j=1}^{m}\left|x_{j}-y_{j}\right|+\sum_{j=m+1}^{n}\left|y_{j}-x_{j}-A^{j}(x) \cdot(y-x)\right|^{\frac{1}{2}} \\
\leq & \sum_{j=1}^{m}\left|x_{j}-y_{j}\right|+\sum_{j=m+1}^{n}\left|y_{j}-x_{j}-A^{j}(y) \cdot(y-x)\right|^{\frac{1}{2}} \\
& +\sum_{j=m+1}^{n}\left|\left(A^{j}(x)-A^{j}(y)\right) \cdot(y-x)\right|^{\frac{1}{2}} \\
\leq & \rho(y, x)+c|x-y| \\
\leq & c \rho(y, x)
\end{aligned}
$$

by (19). The "triangle inequality" can be proved as follows:

$$
\begin{aligned}
\rho(x, y)= & \sum_{j=1}^{m}\left|x_{j}-y_{j}\right|+\sum_{j=m+1}^{n}\left|y_{j}-x_{j}-A^{j}(x) \cdot(y-x)\right|^{\frac{1}{2}} \\
\leq & \sum_{j=1}^{m}\left(\left|y_{j}-z_{j}\right|+\left|z_{j}-x_{j}\right|\right) \\
& +\sum_{j=m+1}^{n}\left\{\left|y_{j}-z_{j}-A^{j}(z) \cdot(y-z)\right|^{\frac{1}{2}}\right. \\
& \left.+\left|z_{j}-x_{j}+A^{j}(z) \cdot(y-z)-A^{j}(x) \cdot(y-x)\right|^{\frac{1}{2}}\right\} \\
= & \rho(z, y)+\sum_{j=1}^{m}\left|z_{j}-x_{j}\right|+\sum_{j=m+1}^{n}\left|z_{j}-x_{j}-A^{j}(x) \cdot(z-x)\right|^{\frac{1}{2}} \\
& +\sum_{j=m+1}^{n} \mid\left(A^{j}(x)-\left.A^{j}(z) \cdot(z-y)\right|^{\frac{1}{2}}\right. \\
\leq & \rho(z, y)+\rho(x, z)+c|x-z|^{\frac{1}{2}}|z-y|^{\frac{1}{2}} \\
\leq & c(\rho(z, y)+\rho(x, z)) .
\end{aligned}
$$

Estimate (20) of the measure can be obtained by the change of variable

$$
y \mapsto \xi, \quad \xi_{j}= \begin{cases}y_{j}-x_{j} & \text { if } 1 \leq j \leq m \\ y_{j}-x_{j}-A^{j}(x) \cdot(y-x) & \text { if } m+1 \leq j \leq n .\end{cases}
$$


The change of variable has Jacobian 1 and thus

$$
|\{y: \rho(x, y)<r\}|=\int_{\rho(x, y)<r} d y=\int_{\sum_{j \leq m}\left|\xi_{j}\right|+\sum_{j>m}\left|\xi_{j}\right|^{\frac{1}{2}}<r} d \xi=c r^{Q}
$$

and the proof is finished

By Proposition 2.1, $\rho$ is equivalent to the symmetric function $\tilde{\rho}(x, y)=$ $\rho(x, y)+\rho(y, x)$ which turns out to be a quasi-distance. The set $\Omega$, equipped with the Lebesgue measure and the quasi-distance $\tilde{\rho}$ is a Homogeneous space, in the sense of Coifman and Weiss. Then the following result holds.

Corollary 2.2. The "fractional integration" operator defined by

$$
T_{\mu} f(x)=\int_{\Omega} \frac{f(y) d y}{\rho(x, y)^{Q-\mu}} \quad(x \in \Omega ; 0<\mu<Q)
$$

satisfies

$$
\begin{aligned}
\left\|T_{\mu} f\right\|_{\frac{p Q}{Q-\mu p}} & \leq c_{5}\|f\|_{p} & & \left(1<p<\frac{Q}{\mu}, f \in L^{p}(\Omega)\right)(21 \\
\sup _{\lambda>0} \lambda\left|\left\{x:\left|T_{\mu} f(x)\right|>\lambda\right\}\right|^{\frac{Q-\mu}{Q}} & \leq c_{6}\|f\|_{L^{1}} & & \left(f \in L^{1}(\Omega)\right) \\
\int_{\Omega} \exp \left(\frac{\left|T_{\mu} f(x)\right|}{c_{7}\|f\|_{\frac{Q}{\mu}}}\right)^{\frac{Q}{Q-\mu}} & \leq c_{8} & & \left(f \in L^{\frac{Q}{\mu}}(\Omega)\right) .
\end{aligned}
$$

Proof. The proof of (21) and (22) is contained in [15], while (23) is proved in $[16]$

Let $\Gamma_{\bar{x}}(x, \cdot)$ be the fundamental solution of the hypoelliptic operator $\sum_{j=1}^{m} X_{j, \bar{x}}^{2}$ with pole at $x$. In the following proposition we give some pointwise estimates concerning $\Gamma_{x}(x, y)$.

Proposition 2.3. Assume that $a_{j}^{k} \in \operatorname{Lip}\left(\mathbb{R}^{n}\right)$ and define $a_{j, x}^{k}$ as in (12). Given an integer $k>0$, denote by $X_{x}^{k}$ any derivative of the form $X_{j_{1}, x} X_{j_{2}, x} \cdots X_{j_{k}, x}\left(j_{i} \in\right.$ $\{1, \ldots, m\})$. Denote also

$$
\left(a_{x}(y)-a(y)\right)^{k}=\prod_{l=1}^{k}\left(a_{j_{l}, x}^{k_{l}}(y)-a_{j_{l}}^{k_{l}}(y)\right)
$$

$\left(j_{i} \in\{1, \ldots, m\}\right.$ and $\left.k_{i} \in\{m+1, \ldots, n\}\right)$. Then there exists a constant $c>0$ such that

$$
\left|X_{x}^{k} \Gamma_{x}(x, y)\right| \leq c \frac{1}{\rho(x, y)^{Q-2+k}} \quad(x, y \in \Omega) .
$$


Moreover, there exists a constant $\varepsilon \in(0,1)$ such that if $x, y, z \in \Omega$ and $\rho(z, x) \leq \varepsilon \rho(x, y)$, then

$$
\left|X_{x}^{k} \Gamma_{x}(x, y)-X_{z}^{k} \Gamma_{z}(z, y)\right| \leq c \rho(z, x)^{\frac{1}{2}} \frac{1}{\rho(x, y)^{Q+k-\frac{3}{2}}}
$$

if $x, y, z \in \Omega$ with $\rho(z, x) \leq \varepsilon \rho(x, y)$.

Remark 2.4. In the smooth case estimate the right-hand side in (25) has the better form $c \frac{\rho(z, x)}{\rho(x, y)^{Q+k-1}}$.

Proof of Proposition 2.3. It is easy to check that the fundamental solution of $\sum_{j=1}^{m} X_{j, \bar{x}}^{2}$ can be written as

$$
\Gamma_{\bar{x}}(x, y)=\Gamma_{H}\left(\psi_{\bar{x}}(x), \psi_{\bar{x}}(y)\right)
$$

where $\Gamma_{H}$ denotes the fundamental solution of the sub-Laplacian $\sum H_{j}^{2}$. In particular, if $x=\bar{x}$, we have

$$
\Gamma_{x}(x, y)=\Gamma_{H}\left(\psi_{x}(x), \psi_{\bar{x}}(y)\right)=\Gamma_{H}\left(0, \psi_{\bar{x}}(y)\right)=\Gamma_{H}\left(\psi_{\bar{x}}(y)\right)
$$

by a slight abuse of notation. Thus

$$
\left|X_{x}^{k} \Gamma_{x}(x, y)\right|=\left|H^{k} \Gamma_{H}\left(\psi_{x}(y)\right)\right| \leq c \frac{1}{\left\|\psi_{x}(y)\right\|^{Q-2+k}} .
$$

The last inequality is a standard estimate concerning the fundamental solution of a sub-Laplacian on a Carnot Group (see [7]). Here we let

$$
\|u\|=\sum_{j=1}^{n}\left|u_{j}\right|+\sum_{j=m+1}^{n}\left|u_{j}\right|^{\frac{1}{2}} .
$$

The function $u \mapsto\|u\|$ is homogeneous of degree 1 with respect to the family of dilations $(D(\lambda))_{\lambda>0}$. Hence in the sequel we will call it homogeneous norm.

Now, by the explicit form (16) of $\psi_{x}$, the proof of (24) can be concluded 
as follows:

$$
\begin{aligned}
\left\|\psi_{x}(y)\right\|= & \| \sum_{j=1}^{m}(y-x)_{j} e_{j} \\
& +\sum_{j=m+1}^{n}\left\{(y-x)_{j}-(y-x) \cdot A^{j}(x)+\frac{1}{2} B^{j}(y-x) \cdot(y-x)\right\} e_{j} \| \\
= & \sum_{j=1}^{m}\left|y_{j}-x_{j}\right| \\
& +\sum_{j=m+1}^{n}\left|(y-x)_{j}-(y-x) \cdot A^{j}(x)+\frac{1}{2} B^{j}(y-x) \cdot(y-x)\right|^{\frac{1}{2}} \\
\simeq & \sum_{j=1}^{m}\left|y_{j}-x_{j}\right|+\sum_{j=m+1}^{n}\left|(y-x)_{j}-(y-x) \cdot A^{j}(x)\right|^{\frac{1}{2}} \\
= & \rho(x, y) .
\end{aligned}
$$

The last equivalence is a consequence of the estimate

$$
\left|B^{j}(y-x) \cdot(y-x)\right|^{\frac{1}{2}} \leq c \sum_{k=1}^{m}\left|y_{k}-x_{k}\right| .
$$

In order to prove (25) we first prove the implication

$$
\rho(x, z) \leq \varepsilon \rho(x, y) \quad \Longrightarrow \quad\left\|\psi_{x}(y)^{-1} \circ \psi_{z}(y)\right\| \leq c \sqrt{\varepsilon}\left\|\psi_{x}(y)\right\|
$$

where the constant $c>0$ does not depend on $x, y \in \Omega$. The group law (17) gives

$$
\begin{aligned}
\psi_{x}(y)^{-1} \circ \psi_{z}(y) & \\
= & \sum_{j=1}^{m}\left[\left(y_{j}-z_{j}\right)-\left(y_{j}-x_{j}\right)\right] e_{j} \\
+ & \sum_{j=m+1}^{n}\left\{\left[y_{j}-z_{j}-(y-z) \cdot A^{j}(z)-\frac{1}{2} B^{j}(y-z) \cdot(y-z)\right] e_{j}\right. \\
& -\left[(y-x)_{j}-(y-x) \cdot A^{j}(x)-\frac{1}{2} B^{j}(y-x) \cdot(y-x)\right] e_{j} \\
& \left.-\frac{1}{2}\left[B^{j}(y-x) \cdot(y-z)-B^{j}(y-z) \cdot(y-x)\right]\right\} e_{j} \\
= & \sum_{j=1}^{m}\left(x_{j}-z_{j}\right) e_{j}+\sum_{j=m+1}^{n}\left\{x_{j}-z_{j}-A^{j}(z) \cdot(y-z)+A^{j}(x) \cdot(y-x)\right. \\
& \left.-\frac{1}{2}\left[B^{j}(y-x) \cdot(x-z)+B^{j}(y-z) \cdot(x-z)\right]\right\} \cdot
\end{aligned}
$$


The homogeneous norm is

$$
\begin{aligned}
\left\|\psi_{x}(y)^{-1} \circ \psi_{z}(y)\right\| & \sum_{j=1}^{m}\left|x_{j}-z_{j}\right|+\sum_{j=m+1}^{n} \mid x_{j}-z_{j}-A^{j}(z) \cdot(y-z)+A^{j}(x) \cdot(y-x) \\
& \quad-\left.\frac{1}{2}\left[B^{j}(y-x) \cdot(x-z)+B^{j}(y-z) \cdot(x-z)\right]\right|^{\frac{1}{2}} \\
\leq & \sum_{j=1}^{m}\left|x_{j}-z_{j}\right|+\sum_{j=m+1}^{n}\left|x_{j}-z_{j}-A^{j}(z) \cdot(x-z)\right|^{\frac{1}{2}} \\
& +\sum_{j=m+1}^{n} \mid\left(A^{j}(z)-A^{j}(x)\right) \cdot(x-y) \\
& \quad-\left.\frac{1}{2}\left[B^{j}(y-x) \cdot(x-z)+B^{j}(y-z) \cdot(x-z)\right]\right|^{\frac{1}{2}} \\
\leq & \rho(z, x)+c(|z-x||x-y|)^{\frac{1}{2}} \\
\leq & c \rho(z, x)+\rho(x, y)^{\frac{1}{2}} \rho(z, x)^{\frac{1}{2}} \\
\leq & c \sqrt{\varepsilon} \rho(x, y)
\end{aligned}
$$

as soon as $\rho(x, z) \leq \varepsilon \rho(x, y)$. Thus (26) is proved. Then, if $\rho(x, z) \leq \varepsilon \rho(x, y)$ and $\varepsilon>0$ is small enough, by (26),(27) and the standard Calderon-Zygmund type estimate for the derivatives of the fundamental solution of sub-Laplacians on Carnot groups, we get

$$
\begin{aligned}
\left|X_{x}^{k} \Gamma_{x}(x, y)-X_{z}^{k} \Gamma_{z}(z, y)\right| & =\left|H^{k} \Gamma_{H}\left(\psi_{x}(y)\right)-H^{k} \Gamma_{H}\left(\psi_{z}(y)\right)\right| \\
& \leq c \frac{\left\|\psi_{x}(y)^{-1} \circ \psi_{z}(y)\right\|}{\left\|\psi_{x}(y)\right\|^{Q+k-1}} .
\end{aligned}
$$

Deleting the last line in (27) we get the estimate

$$
\left\|\psi_{x}(y)^{-1} \circ \psi_{z}(y)\right\| \leq c \rho(x, y)^{\frac{1}{2}} \rho(z, x)^{\frac{1}{2}}
$$

which ends the proof of $(25)$ 


\section{Sobolev and Trudinger inequalities}

In this section we prove part (A) and (B) of Theorem 1.1. The proof will be a consequence of the following proposition.

Proposition 3.1. Let $\Omega \subset \mathbb{R}^{n}$ be a bounded open set. Denote by $\rho$ the function introduced in Proposition 2.1. Then there exists a constant $c>0$ such that for any $f \in C_{0}^{\infty}(\Omega)$ the estimate

$$
|f(x)| \leq c \int_{\Omega} \frac{|X f(y)|}{\rho(x, y)^{Q-1}} d y
$$

holds.

Proof. Representing the function $f \in C_{0}^{\infty}(\Omega)$ by means of the fundamental solution $\Gamma_{x}(x, y)$ of the operator $\sum_{j=1}^{m} X_{j, x}^{2}$ with pole at $x$ we have

$$
\begin{aligned}
f(x)= & \sum_{j=1}^{m} \int_{\Omega} X_{j, x} \Gamma_{x}(x, y) X_{j, x} f(y) d y \\
= & \sum_{j=1}^{m} \int_{\Omega} X_{j, x} \Gamma_{x}(x, y)\left(X_{j, x}-X_{j}+X_{j}\right) f(y) d y \\
= & \sum_{j=1}^{m} \int_{\Omega} X_{j, x} \Gamma_{x}(x, y) \sum_{k=m+1}^{n}\left(a_{j, x}^{k}(y)-a_{j}^{k}(y)\right) \frac{\partial}{\partial y_{k}} f(y) d y \\
& +\sum_{j=1}^{m} \int_{\Omega} X_{j, x} \Gamma_{x}(x, y) X_{j} f(y) d y \\
= & : I_{1}(x)+I_{1}^{\prime}(x) .
\end{aligned}
$$

Proposition 2.3 gives the desired estimate of $I_{1}^{\prime}$

$$
\left|I_{1}^{\prime}(x)\right| \leq c \int \frac{|X f(y)|}{\rho(x, y)^{Q-1}} d y .
$$

Rewrite now (7) in the form

$$
\partial_{y_{k}}=\sum_{i, l=1}^{m} \tilde{\lambda}_{k}^{i, l}(y) X_{i} X_{l}
$$

where we let $\tilde{\lambda}_{k}^{i, l}=\lambda_{k}^{i, l}$ if $i<l, \tilde{\lambda}_{k}^{i, l}=-\lambda_{k}^{l, i}$ if $i>l$ and $\tilde{\lambda}_{k}^{i, i}=0$. The functions $\lambda_{k}^{i, l}$ also satisfy hypothesis (8). Thus the evaluation of $I_{1}$ will be ensured by the estimate of the integral

$$
J(x)=\int X_{j, x} \Gamma_{x}(x, y)\left(a_{j, x}^{k}(y)-a_{j}^{k}(y)\right) \tilde{\lambda}_{k}^{i, l}(y) X_{i} X_{l} f(y) d y
$$


$(i, j, l=1, \ldots, m$ and $k=m+1, \ldots, n)$. We now integrate by part in (30). In order to deal correctly with the singular terms we first truncate the domain of integration. Write for brevity

$$
g(x, y)=X_{j, x} \Gamma_{x}(x, y)\left(a_{j, x}^{k}(y)-a_{j}^{k}(y)\right) \tilde{\lambda}_{k}^{i, l}(y) .
$$

Since by (24) and (20) the function $y \mapsto X_{j, x} \Gamma_{x}(x, y)$ is integrable in $\Omega$ and all the remaining functions appearing in integral (30) are bounded we have

$$
J(x)=\lim _{\varepsilon \rightarrow 0} J_{\varepsilon}(x) \quad \text { where } \quad J_{\varepsilon}(x)=\int_{\Omega_{\varepsilon}} g(x, y) X_{i} X_{l} f(y) d y
$$

and

$$
\Omega_{\varepsilon}=\Omega \backslash\left\{y: \Gamma_{x}(x, y)>\varepsilon^{2-Q}\right\} .
$$

Recall that the functions $\tilde{\lambda}_{k}^{i, l}$ are bounded together with their distributional derivatives $X_{i} \tilde{\lambda}_{k}^{i, l}$. Then, by the results of $[12,14]$, there exists a family $\left(\tilde{\lambda}_{k}^{i, l}\right)_{\sigma} \quad(\sigma>0)$ of smooth functions on $\Omega$ such that $\left(\tilde{\lambda}_{k}^{i, l}\right)_{\sigma} \rightarrow \tilde{\lambda}_{k}^{i, l}$ and $X_{i}\left(\lambda_{k}^{i, l}\right)_{\sigma} \rightarrow X_{i} \tilde{\lambda}_{k}^{i, l}$ in $L^{2}(K)$ as $\sigma \rightarrow 0$, where $K$ is the support of $f$. Approximating the functions $\tilde{\lambda}_{k}^{i, l}$ in the described way, we can apply on the set $\Omega_{\varepsilon}$ the integration by part formula

$$
\begin{aligned}
\int_{\Omega_{\varepsilon}} & g(y) X v(y) d y \\
\quad & =\int_{\partial \Omega_{\varepsilon}} g(y) v(y) X(y) \cdot \nu(y) d \sigma(y)-\int_{\Omega_{\varepsilon}}(X g(y)+\operatorname{div} X(y) g(y)) v(y) d y
\end{aligned}
$$

where $v$ and $g$ are Lipschitz-continuous functions, $X=\sum_{j=1}^{n} a^{j} \partial_{j}$ is a Lipschitzcontinuous vector field, $\operatorname{div} X(y)=\sum_{j=1}^{n} \partial_{j} a^{j}(y), \nu(y)$ is the outer normal to $\Omega_{\varepsilon}$ at the point $y, d \sigma$ denotes the surface measure and $X(y) \cdot \nu(y)=$ $\sum_{j=1}^{n} a_{j}(y) \nu_{j}(y)$. Thus

$$
\begin{aligned}
J_{\varepsilon}(x) & =\int_{\partial \Omega_{\varepsilon}} g(x, y) X_{l} f(y) X_{i}(y) \cdot \nu(y) d \sigma(y)-\int_{\Omega_{\varepsilon}}\left(X_{i}+\operatorname{div} X_{i}\right) g(x, y) X_{l} f(y) d y \\
& =: J_{\varepsilon}^{\prime}(x)+J_{\varepsilon}^{\prime \prime}(x)
\end{aligned}
$$

We first show that $J_{\varepsilon}^{\prime}(x) \rightarrow 0$ as $\varepsilon \rightarrow 0$. Denote by $\nabla$ the Euclidean gradient. Then

$$
X_{i}(y) \cdot \nu(y)=X_{i}(y) \cdot \frac{\nabla \Gamma_{x}(x, y)}{\left|\nabla \Gamma_{x}(x, y)\right|}=\frac{X_{i} \Gamma_{x}(x, y)}{\left|\nabla \Gamma_{x}(x, y)\right|} .
$$


Moreover, by also using (14) and (10),

$$
\begin{aligned}
X_{i} \Gamma_{x}(x, y) & =\left(X_{i}-X_{i, x}\right) \Gamma_{x}(x, y)+X_{i, x} \Gamma_{x}(x, y) \\
& =\sum_{k=m+1}^{n}\left(a_{i}^{k}-a_{i, x}^{k}\right)(y) \partial_{y_{k}} \Gamma_{x}(x, y)+X_{i, x} \Gamma_{x}(x, y) \\
& =\sum_{k=m+1}^{n}\left(a_{i}^{k}-a_{i, x}^{k}\right)(y)\left[X_{g_{1}(k), x}, X_{g_{2}(k), x}\right] \Gamma_{x}(x, y)+X_{i, x} \Gamma_{x}(x, y),
\end{aligned}
$$

hence

$$
\begin{aligned}
& \left|g(x, y) X_{i} \Gamma_{x}(x, y)\right| \\
& \leq c \rho(x, y)\left|X_{j, x} \Gamma_{x}(x, y)\right| \\
& \quad \times\left(\left|X_{i, x} \Gamma_{x}(x, y)\right|+\rho(x, y) \sum_{k=m+1}^{n}\left|\left[X_{g_{1}(k), x}, X_{g_{2}(k), x}\right] \Gamma_{x}(x, y)\right|\right) \\
& \leq c(\rho(x, y))^{3-2 Q} .
\end{aligned}
$$

The function $X_{l} f$ is bounded on $\Omega$ and vanishes on $\partial \Omega$. Thus, for any fixed $x$ we have

$$
\begin{aligned}
\left|J_{\varepsilon}^{\prime}(x)\right| & =\left|\int_{\left\{\Gamma_{x}(x, y)=\varepsilon^{2-Q}\right\}} g(x, y) X_{l} f(y) X_{i}(y) \cdot \nu(y) d \sigma(y)\right| \\
& \leq \frac{c}{\varepsilon^{2 Q-3}} \int_{\left\{\Gamma_{x}(x, y)=\varepsilon^{2-Q}\right\}} \frac{1}{\left|\nabla \Gamma_{x}(x, y)\right|} d \sigma(y) .
\end{aligned}
$$

Define

$$
F(r)=\frac{1}{r^{Q-1}} \int_{\left\{\Gamma_{x}(x, y)=r^{2-Q}\right\}} \frac{1}{\left|\nabla \Gamma_{x}(x, y)\right|} d \sigma(y)
$$

Then, by the coarea formula [5: p. 118] with $f(y)=\left(\Gamma_{x}(x, y)\right)^{\frac{1}{2-Q}}$ and by the change of variables (16), we get

$$
\begin{aligned}
\int_{0}^{\varepsilon} F(r) d r & =(Q-2) \int_{0}^{\varepsilon}\left(\int_{\{r=f(y)\}} \frac{1}{|\nabla f(y)|} d \sigma(y)\right) d r \\
& =(Q-2) \int_{\left\{\Gamma_{x}(x, y)>\varepsilon^{2-Q}\right\}} d y \\
& =(Q-2) \int_{\left\{\Gamma_{H}(\xi)>\varepsilon^{2-Q}\right\}} d \xi \\
& =(Q-2) \varepsilon^{Q} \int_{\left\{\Gamma_{H}(\xi)>1\right\}} d \xi
\end{aligned}
$$


Hence, by differentiating the previous equality with respect to $\varepsilon$, we get $F(\varepsilon)=$ $c \varepsilon^{Q-1}$ and

$$
\left|J_{\varepsilon}^{\prime}(x)\right| \leq \frac{c}{\varepsilon^{Q-2}} F(\varepsilon)=c \varepsilon .
$$

The last term tends to zero as $\varepsilon \rightarrow 0$.

In order to rewrite $J_{\varepsilon}^{\prime \prime}=J_{\varepsilon}^{\prime \prime}(x)$ we use the explicit form (31) of $g$ :

$$
\begin{aligned}
J_{\varepsilon}^{\prime \prime}= & -\int_{\Omega_{\varepsilon}} X_{i}\left[X_{j, x} \Gamma_{x}(x, y)\left(a_{j, x}^{k}(y)-a_{j}^{k}(y)\right) \tilde{\lambda}_{j}^{i, l}(y)\right] X_{l} f(y) d y \\
& -\int_{\Omega_{\varepsilon}} \operatorname{div} X_{i}(y)\left[X_{j, x} \Gamma_{x}(x, y)\left(a_{j, x}^{k}(y)-a_{j}^{k}(y)\right) \tilde{\lambda}_{j}^{i, l}(y)\right] X_{l} f(y) d y \\
= & -\int_{\Omega_{\varepsilon}} X_{i} X_{j, x} \Gamma_{x}(x, y)\left(a_{j, x}^{k}(y)-a_{j}^{k}(y)\right) \tilde{\lambda}_{j}^{i, l}(y) X_{l} f(y) d y \\
& -\int_{\Omega_{\varepsilon}} X_{j, x} \Gamma_{x}(x, y)\left(X_{i} a_{j}^{k}(y)-X_{i} a_{j, x}^{k}(y)\right) \tilde{\lambda}_{j}^{i, l}(y) X_{l} f(y) d y \\
& -\int_{\Omega_{\varepsilon}} X_{j, x} \Gamma_{x}(x, y)\left(a_{j, x}^{k}(y)-a_{j}^{k}(y)\right) X_{i} \tilde{\lambda}_{j}^{i, l}(y) X_{l} f(y) d y \\
& -\int_{\Omega_{\varepsilon}} \operatorname{div} X_{i}(y)\left[X_{j, x} \Gamma_{x}(x, y)\left(a_{j, x}^{k}(y)-a_{j}^{k}(y)\right) \tilde{\lambda}_{j}^{i, l}(y)\right] X_{h} f(y) d y \\
= & B_{\varepsilon}(x)+B_{1, \varepsilon}(x)+B_{2, \varepsilon}(x)+B_{3, \varepsilon}(x) .
\end{aligned}
$$

Rewrite the term $B_{\varepsilon}$ using $X_{i}=X_{i}-X_{i, x}+X_{i, x}$ :

$$
\begin{aligned}
B_{\varepsilon}(x)= & \sum_{h=m+1}^{n} \int_{\Omega_{\varepsilon}}\left(a_{i}^{h}(y)-a_{i, x}^{h}(y)\right) \\
& \times \partial_{y_{h}} X_{j, x} \Gamma(x, y)\left(a_{j, x}^{k}(y)-a_{j}^{k}(y)\right) \tilde{\lambda}_{k}^{i, l}(y) X_{l} f(y) d y \\
& +\int_{\Omega_{\varepsilon}} X_{i, x} X_{j, x} \Gamma(x, y)\left(a_{j, x}^{k}(y)-a_{j}^{k}(y)\right) \tilde{\lambda}_{k}^{i, h}(y) X_{l} f(y) d y \\
= & : B_{4, \varepsilon}(x)+B_{5, \varepsilon}(x) .
\end{aligned}
$$

We now show that each of the integrals $B_{k, 0}(x) \quad(k=1, \ldots, 5)$ is well defined. This guarantees that $B_{k, 0}(x)=\lim _{\varepsilon \rightarrow 0} B_{k, \varepsilon}(x)$. More precisely, we will show that $\left|B_{k, 0}(x)\right| \leq T_{1}(|X f|)(x)$.

Since $\tilde{\lambda}_{k}^{i, h} \in L^{\infty}$ and $\left|X_{i} a_{j, x}^{k}\right| \leq c$, we see that

$$
\left|B_{1,0}(x)\right| \leq c \int_{\Omega}\left|X_{j, x} \Gamma(x, y)\right|\left|X_{l} f(y)\right| d y .
$$

Then the term $B_{1,0}$ can be estimated using the same arguments of $I_{1}^{\prime}$ (see (28)). The estimate of the term $B_{2,0}$ can be done using the Lipschitz continuity of 
the fields (which, by also (19), gives $\left|a_{j, x}^{k}(y)-a_{j}^{k}(y)\right| \leq c|x-y| \leq c \rho(x, y)$ ) and the boundedness of $X_{i} \tilde{\lambda}_{k}^{i, h}$. Then

$$
\begin{aligned}
\left|B_{2,0}(x)\right| & \leq c \int_{\Omega}\left|X_{j, x} \Gamma_{x}(x, y)\right| \rho(x, y)\left|X_{i} \tilde{\lambda}_{k}^{i, h}(y)\right|\left|X_{l} f(y)\right| d y \\
& \leq c T_{2}(|X f|)(x) \\
& \leq c T_{1}(|X f|)(x) .
\end{aligned}
$$

The term $B_{3,0}$ can be evaluated with an argument similar to the one used for $B_{2,0}$. By (14) and (10) the term $B_{4,0}$ is controlled by an expression of the form

$$
\left|B_{4,0}(x)\right| \leq c \int_{\Omega}\left|X_{x}^{3} \Gamma_{x}(x, y)\left(a_{x}-a\right)^{2}(y)\right| X f(y) \mid d y \leq c T_{1}(|X f|)(x)
$$

We used here the notations introduced in the statement of Proposition 2.3. Finally, the estimate of $B_{5,0}$ can be obtained with a similar argument

Proof of the Sobolev and Trudinger inequalities. The proof of the Sobolev inequality for $1<p<Q$ and the Trudinger inequality follows from the "representation formula" proved in the Proposition 3.1 and from the continuity estimates of Corollary 2.2. In the case $p=1$ Corollary 2.2 provides the weak inequality

$$
\sup _{\lambda>0} \lambda|\{|f|>\lambda\}|^{\frac{Q-1}{Q}} \leq c\|X f\|_{L^{1}}
$$

which, by the truncation technique used in [9: p. 564], can be improved to the desired estimate $\left(\int_{\Omega}|f|^{\frac{Q}{Q-1}}\right)^{\frac{Q-1}{Q}} \leq c \int_{\Omega}|X f|$

\section{Morrey estimate and compact embedding}

In the following proposition we give an estimate of the difference between the values of a function $f$ at two different points in term of integrals involving the degenerate gradient of $f$ and suitable kernels. This will enable us to prove the Morrey estimate $(\mathrm{C})$ in Theorem 1.1 and a fractional-type estimate which will provide the compact embedding (D) in Theorem 1.1.

Proposition 4.1. Let $f \in C_{0}^{\infty}(\Omega)$. Then

$$
|f(x)-f(z)| \leq c \int_{\Omega} K(x, z ; y)|X f(y)| d y \quad(x, z \in \Omega)
$$


where the kernel $K$ is a sum of a finite number of terms of the form

$$
\begin{aligned}
& R_{k}(x, z ; y)=\left|X_{x}^{k+1} \Gamma_{x}(x, y)\left(a_{x}(y)-a(y)\right)^{k}-X_{z}^{k+1} \Gamma_{z}(z, y)\left(a_{z}(y)-a(y)\right)^{k}\right| \\
& (k=0,1,2) \text { and } \\
& \quad R(x, z ; y)=\left|X_{x} \Gamma_{x}(x, y)\left(a_{x}(y)-a(y)\right)-X_{z} \Gamma_{z}(z, y)\left(a_{z}(y)-a(y)\right)\right| \cdot
\end{aligned}
$$

In (32) and (33) we used the notations of Proposition 2.3. Moreover, if $\rho(x, z) \leq \varepsilon \rho(x, y)$, then

$$
K(x, y ; z) \leq c \frac{\rho(z, x)^{\frac{1}{2}}}{\rho(x, y)^{Q-\frac{1}{2}}} .
$$

Proof. For $x, z \in \Omega$ and $f \in C_{0}^{\infty}$ write $f(x)$ and $f(z)$ as in (28). Then

$$
f(x)-f(z)=\left(I_{1}(x)-I_{1}(z)\right)+\left(I_{1}^{\prime}(x)-I_{1}^{\prime}(z)\right) .
$$

The second term $I_{1}^{\prime}(x)-I_{1}^{\prime}(z)$ has the appropriate form, since

$$
\left|I_{1}^{\prime}(x)-I_{1}^{\prime}(z)\right| \leq \sum_{j=1}^{m} \int_{\Omega}\left|X_{j, x} \Gamma_{x}(x, y)-X_{j, z} \Gamma_{z}(z, y)\right|\left|X_{j} f(y)\right| d y
$$

and the kernel $\left|X_{j, x} \Gamma_{x}(x, y)-X_{j, z} \Gamma_{z}(z, y)\right|$ is of type (32) with $k=0$. Now we will integrate by part the first term $I_{1}(x)-I_{1}(z)$ by using (29):

$$
\begin{aligned}
I_{1}(x)-I_{1}(z)= & \sum_{j=1}^{m} \sum_{k=m+1}^{n} \int\left\{X_{j, x} \Gamma_{x}(x, y)\left(a_{j, x}^{k}-a_{j}^{k}\right)(y)\right. \\
& \left.-X_{j, z} \Gamma_{z}(x, y)\left(a_{j, z}^{k}-a_{j}^{k}\right)(y)\right\} \partial_{y_{k}} f(y) d y \\
= & \sum_{j=1}^{m} \sum_{k=m+1}^{n} \sum_{i, l=1}^{m} \int\left\{X_{j, x} \Gamma_{x}(x, y)\left(a_{j, x}^{k}-a_{j}^{k}\right)(y)\right. \\
& \left.-X_{j, z} \Gamma_{z}(x, y)\left(a_{j, z}^{k}-a_{j}^{k}\right)(y)\right\} \tilde{\lambda}_{k}^{i, l}(y) X_{i} X_{l} f(y) d y \\
= & \int S(x, z ; y) X_{l} f(y) d y
\end{aligned}
$$

where the kernel $S$ is defined by the last equality through an integration by part. Denote $X_{i}^{*}=-X_{i}-\operatorname{div}\left(X_{i}\right)$ and write for brevity $\Gamma_{x}$ instead of 
$\Gamma_{x}(x, y)$. By omitting the summation symbol on the indices $j, i, k$ and $l$, the kernel $S=S(x, z ; y)$ has the following form (take also (13) into account):

$$
\begin{aligned}
S= & X_{i}^{*}\left\{\left(X_{j, x} \Gamma_{x}\left(a_{j, x}^{k}-a_{j}^{k}\right)(y)-X_{j, z} \Gamma_{z}\left(a_{j, z}^{k}-a_{j}^{k}\right)(y)\right) \tilde{\lambda}_{k}^{i, l}(y)\right\} \\
= & -\left\{X_{j, x} \Gamma_{x}\left(X_{i} a_{j, x}^{k}-X_{i} a_{j}^{k}\right)(y)-X_{j, z} \Gamma_{z}\left(X_{i} a_{j, z}^{k}-X_{i} a_{j}^{k}\right)(y)\right\} \tilde{\lambda}_{k}^{i, l}(y) \\
& -\left\{X_{i} X_{j, x} \Gamma_{x}\left(a_{j, x}^{k}-a_{j}^{k}\right)(y)-X_{i} X_{j, z} \Gamma_{z}\left(a_{j, z}^{k}-a_{j}^{k}\right)(y)\right\} \tilde{\lambda}_{k}^{i, l}(y) \\
& -\left\{X_{j, x} \Gamma_{x}\left(a_{j, x}^{k}-a_{j}^{k}\right)(y)-X_{j, z} \Gamma_{z}\left(a_{j, z}^{k}-a_{j}^{k}\right)(y)\right\} X_{i} \tilde{\lambda}_{k}^{i, l}(y) \\
& -\left(X_{j, x} \Gamma_{x}\left(a_{j, x}^{k}-a_{j}^{k}\right)(y)-X_{j, z} \Gamma_{z}\left(a_{j, z}^{k}-a_{j}^{k}\right)(y)\right) \operatorname{div} X_{i}(y) \tilde{\lambda}_{k}^{i, l}(y) \\
=- & \left\{\left(X_{j, x} \Gamma_{x}-X_{j, z} \Gamma_{z}\right)\left(X_{i} a_{j, x}^{k}-X_{i} a_{j}^{k}\right)(y)\right\} \tilde{\lambda}_{k}^{i, l}(y) \\
- & \left\{\left(X_{i}-X_{i, x}\right) X_{j, x} \Gamma_{x}\left(a_{j, x}^{k}-a_{j}^{k}\right)(y)-\left(X_{i}-X_{i, z}\right) X_{j, z} \Gamma_{z}\left(a_{j, z}^{k}-a_{j}^{k}\right)(y)\right\} \tilde{\lambda}_{k}^{i, l}(y) \\
- & \left\{X_{i, x} X_{j, x} \Gamma_{x}\left(a_{j, x}^{k}-a_{j}^{k}\right)(y)-X_{i, z} X_{j, z} \Gamma_{z}\left(a_{j, z}^{k}-a_{j}^{k}\right)(y)\right\} \tilde{\lambda}_{k}^{i, l}(y) \\
- & \left\{X_{j, x} \Gamma_{x}\left(a_{j, x}^{k}-a_{j}^{k}\right)(y)-X_{j, z} \Gamma_{z}\left(a_{j, z}^{k}-a_{j}^{k}\right)(y)\right\} X_{i} \tilde{\lambda}_{k}^{i, l}(y) \\
- & \left\{X_{j, x} \Gamma_{x}\left(a_{j, x}^{k}-a_{j}^{k}\right)(y)-X_{j, z} \Gamma_{z}\left(a_{j, z}^{k}-a_{j}^{k}\right)(y)\right\} \operatorname{div} X_{i}(y) \\
=: & -A_{1}-A_{2}-A_{3}-A_{4}-A_{5} .
\end{aligned}
$$

Recall that, by (14) and (10), for any $\xi \in \mathbb{R}^{n}$ and $s=m+1, \ldots, n$,

$$
\frac{\partial}{\partial y_{s}}=\left[X_{g_{1}(s), \xi}, X_{g_{2}(s), \xi}\right]
$$

Write this last equality with $\xi=x$ and $\xi=z$. Thus

$$
\begin{aligned}
A_{2}= & \sum_{s=m+1}^{n}\left\{\left(a_{i}^{s}-a_{i, x}^{s}\right)(y)\left[X_{g_{1}(s), x}, X_{g_{2}(s), x}\right] X_{j, x} \Gamma_{x}\left(a_{j, x}^{k}-a_{j}^{k}\right)(y)\right. \\
& \left.-\left(a_{i}^{s}-a_{i, z}^{s}\right)(y)\left[X_{g_{1}(s), z}, X_{g_{2}(s), z}\right] X_{j, z} \Gamma_{z}\left(a_{j, z}^{k}-a_{j}^{k}\right)(y)\right\} \tilde{\lambda}_{k}^{i, l}(y) .
\end{aligned}
$$

Then each of the terms $\left|A_{1}\right|,\left|A_{2}\right|,\left|A_{3}\right|$ can be estimated (using $\tilde{\lambda}_{j}^{i, l} \in L^{\infty}$ ) by a kernel of the form $R_{k}(x, z ; y)$ in (32) with $k=0,1,2$.

The terms $\left|A_{4}\right|$ and $\left|A_{5}\right|$ can be estimated (using $X_{j} \tilde{\lambda}_{k}^{i, l}, \operatorname{div} X_{j} \in L^{\infty}$ ) by the less singular kernel $R$ defined in (33). The estimate of the kernel $R_{k}$ can 
be obtained by means of (25):

$$
\begin{aligned}
R_{k}(x, z ; y) \leq & \left|X_{x}^{k+1} \Gamma_{x}(x, y)-X_{z}^{k+1} \Gamma_{z}(z, y)\right|\left|\left(a_{x}(y)-a(y)\right)^{k}\right| \\
& +\left|X_{z}^{k+1} \Gamma_{z}(z, y)\right|\left|\left(a_{x}(y)-a(y)\right)^{k}-\left(a_{z}(y)-a(y)\right)^{k}\right| \\
\leq & c \frac{\rho(z, x)^{\frac{1}{2}}}{\rho(x, y)^{Q+k-\frac{1}{2}}} \rho(x, y)^{k} \\
& +c \frac{1}{\rho(z, y)^{Q+k-1}}\left|\left(a_{x}(y)-a(y)\right)^{k}-\left(a_{z}(y)-a(y)\right)^{k}\right| .
\end{aligned}
$$

The estimate of the last line is non-trivial only if $k=2$. By (12) and (13),

$$
\begin{aligned}
\mid\left(a_{x}(y)\right. & -a(y))^{2}-\left(a_{z}(y)-a(y)\right)^{2} \mid \\
= & \left|\left(a_{j_{1}, x}^{k_{1}}-a_{j_{1}}^{k_{1}}\right)(y)\left(a_{j_{2}, x}^{k_{2}}-a_{j_{2}}^{k_{2}}\right)(y)-\left(a_{j_{1}, z}^{k_{1}}-a_{j_{1}}^{k_{1}}\right)(y)\left(a_{j_{2}, z}^{k_{2}}-a_{j_{2}}^{k_{2}}\right)(y)\right| \\
\leq & \left|\left\{\left(a_{j_{1}, x}^{k_{1}}-a_{j_{1}}^{k_{1}}\right)(y)-\left(a_{j_{1}, z}^{k_{1}}-a_{j_{1}}^{k_{1}}\right)(y)\right\}\left(a_{j_{2}, z}^{k_{2}}-a_{j_{2}}^{k_{2}}\right)(y)\right| \\
& +\left|\left(a_{j_{1}, z}^{k_{1}}-a_{j_{1}}^{k_{1}}\right)(y)\left\{\left(a_{j_{2}, z}^{k_{2}}-a_{j_{2}}^{k_{2}}\right)(y)-\left(a_{j_{2}, x}^{k_{2}}-a_{j_{2}}^{k_{2}}\right)(y)\right\}\right| \\
\leq & c|z-y||z-x| .
\end{aligned}
$$

and the proof is finished

Proof of the Morrey estimate. Let $f \in C_{0}^{\infty}(\Omega)$. Write

$$
I_{k}(x, z)=\int_{\Omega} R_{k}(x, z ; y)|X f(y)| d y
$$

Starting from Proposition 4.1 estimate (C) of Theorem 1.1 will be proved as soon as we will be able to show that $\left|I_{k}(x, z)\right| \leq c \rho^{\alpha}(x, z)$. Fix $\varepsilon>0$ and write

$$
\begin{aligned}
I_{k}(x, z) & =\int_{\rho(x, z) \geq \varepsilon \rho(x, y)} R_{k}(x, z ; y)|X f(y)| d y+\int_{\rho(x, z) \leq \varepsilon \rho(x, y)} R_{k}(x, z ; y)|X f(y)| d y \\
& =: I_{k}^{\prime}(x, z)+I_{k}^{\prime \prime}(x, z)
\end{aligned}
$$

The estimate of $I_{k}^{\prime}$ is easy. Indeed, by (24) and the Lipschitz continuity of the $a_{j}^{k}$ 's we can estimate $I_{k}^{\prime}$ by the Hölder inequality as

$$
I_{k}^{\prime} \leq c\|X f\|_{L^{p}}\left(\int_{\rho(x, z) \geq \varepsilon \rho(x, y)} \frac{d y}{\rho(x, y)^{\frac{(Q-1) p}{p-1}}}+\int_{\rho(x, z) \geq \varepsilon \rho(x, y)} \frac{d y}{\rho(z, y)^{\frac{(Q-1) p}{p-1}}}\right)^{\frac{p-1}{p}}
$$


Both the integrals herein are well defined (the function $\frac{1}{\rho^{\beta}}$ is integrable near 0 if and only if $\beta<Q$ and $\left.p>Q \Leftrightarrow \frac{(Q-1) p}{p-1}<Q\right)$. By the triangle inequality,

$$
\rho(z, y) \leq c(\rho(z, x)+\rho(x, y)) \leq c \rho(z, x)
$$

on the integration set. Then $\{\rho(x, z)>\varepsilon \rho(x, y)\}$ is included in $\{\rho(x, z) \geq$ $c \rho(z, y)\}$. Thus the second integral in (37) can be estimated by the first one. Using polar coordinates we have

$$
\begin{aligned}
I_{k}^{\prime}(x, z) & \leq c\|X f\|_{L^{p}}\left(\int_{\rho(x, y) \leq c \rho(x, z)} \frac{d y}{\rho(x, y)^{\frac{(Q-1) p}{p-1}}}\right)^{\frac{p-1}{p}} \\
& \leq c\|X f\|_{L^{p}}\left(\int_{0}^{c \rho(x, z)} \frac{t^{Q-1} d t}{t^{\frac{(Q-1) p}{p-1}}}\right)^{\frac{p-1}{p}} \\
& =c\|X f\|_{L^{p}} \rho(x, z)^{1-\frac{Q}{p}} .
\end{aligned}
$$

By estimate (34) and the Hölder inequality we get

$$
I_{k}^{\prime \prime} \leq c\|X f\|_{L^{p}} \rho(x, z)^{\frac{1}{2}}\left(\int_{\rho(x, z) \leq \varepsilon \rho(x, y)} \frac{d y}{\rho(x, y)^{\frac{\left(Q-\frac{1}{2}\right) p}{p-1}}}\right)^{\frac{p-1}{p}} .
$$

Using polar coordinates we have

$$
I_{k}^{\prime \prime}(x, z) \leq c \rho(x, z)^{\frac{1}{2}}\|X f\|_{L^{p}}\left(\int_{c \rho(x, z)}^{M} \frac{t^{Q-1} d t}{t^{\frac{\left(Q-\frac{1}{2}\right) p}{p-1}}}\right)^{\frac{p-1}{p}} \leq c\|X f\|_{L^{p}} \rho(x, z)^{\alpha}
$$

with $\alpha=\min \left\{\frac{1}{2}, 1-\frac{Q}{p}\right\}$

Proof of the compact embedding. We prove that for every $1<p<Q$ and $q<\frac{Q p}{Q-p}$ there exists $s>0$ such that the inequality

$$
\left(\int_{\Omega \times \Omega} \frac{|f(x)-f(z)|^{q}}{|x-z|^{n+q s}} d x d z\right)^{\frac{1}{q}} \leq c\left(\int_{\Omega}|X f(x)|^{p} d x\right)^{\frac{1}{p}} .
$$

holds. This, together with the classical compact embedding $W_{0}^{s, q}(\Omega) \subset L^{q}(\Omega)$ will prove the claim.

Let $\bar{s}=\bar{s}(p, q) \in(0,1)$ be the solution of

$$
\frac{p Q}{Q-(1-\bar{s}) p}=q, \quad \text { i.e. } \quad \bar{s}=1-\frac{q-p}{p q} Q .
$$


This choice of $\bar{s}$ ensures that $\left\|T_{1-\bar{s}} f\right\|_{q} \leq c\|f\|_{p}$ for $f \in L^{p}$ (by (21) with $\mu=1-\bar{s})$. Fix now $s<\min \left\{\frac{\bar{s}}{3}, \frac{1}{8}\right\}$ and estimate the fractional norm of a function $f$ as follows: by Proposition 4.1, $|f(x)-f(z)|$ is less than a sum of a finite number of terms of the form $\int_{\Omega} R_{k}(x, z ; y)|X f(y)| d y$, with $R_{k}$ as in (32), plus a finite number of less singular terms which we will not take into account in the sequel. Thus it suffices to evaluate the integral

$$
J=\int_{\Omega \times \Omega} \frac{1}{|x-z|^{n+q s}}\left|\int R_{k}(x, z ; y)\right| X f(y)|d y|^{q} d x d z .
$$

For any integral of this form we can assume by symmetry that the integration takes place over the set where

$$
\left|X_{x}^{k+1} \Gamma_{x}(x, y)\left(a_{x}-a\right)^{k}(y)\right| \geq\left|X_{z}^{k+1} \Gamma_{z}(z, y)\left(a_{z}-a\right)^{k}(y)\right|
$$

The change of variable $z=x+h$ and an application of the Minkowski inequality give

$$
\begin{aligned}
J & \leq c \int_{\Omega} \int_{\{h: x+h \in \Omega\}} \frac{1}{|h|^{n+q s}}\left|\int_{\Omega} R_{k}(x, x+h ; y)\right| X f(y)|d y|^{q} d h d x \\
& \leq c \int_{\Omega}\left[\int_{\Omega}|X f(y)|\left\{\int_{A_{x, y}} \frac{R_{k}(x, x+h ; y)^{q}}{|h|^{n+q s}} d h\right\}^{\frac{1}{q}} d y\right]^{q} d x
\end{aligned}
$$

where we let $A_{x, y}=\{h: x+h \in \Omega$ and (41) holds $\}$. Now write (for a suitably small $\varepsilon>0$ )

$$
\begin{aligned}
\int_{A_{x, y}} & \frac{R_{k}(x, x+h ; y)^{q}}{|h|^{n+q s}} d h \\
= & \int_{\{\rho(x, x+h) \geq \varepsilon \rho(x, y)\} \cap A_{x, y}} \frac{R_{k}(x, x+h ; y)^{q}}{|h|^{n+q s}} d h \\
& +\int_{\{\rho(x, x+h) \leq \varepsilon \rho(x, y)\} \cap A_{x, y}} \frac{R_{k}(x, x+h ; y)^{q}}{|h|^{n+q s}} d h \\
= & : I_{1}+I_{2} .
\end{aligned}
$$

By $(41), R_{k}(x, x+h ; y) \leq \frac{c}{\rho(x, y)^{Q-1}}$ and the inequality $\rho(x, x+h) \leq c|h|^{\frac{1}{2}}$ furnishes

$I_{1} \leq c \int_{|h|^{\frac{1}{2}} \geq \varepsilon \rho(x, y)} \frac{d h}{|h|^{n+q s} \rho(x, y)^{(Q-1) q}}=\frac{c}{\rho(x, y)^{(Q-1+2 s) q}} \leq \frac{c}{\rho(x, y)^{(Q-(1-\bar{s})) q}}$ 
because $2 s<\bar{s}$. The estimate of $I_{2}$ follows from the estimates of $R_{k}$ given in Proposition 4.1. More precisely (recall also that, since $s<\frac{1}{8}, \frac{q}{2}-4 q s>0$ ),

$$
\begin{aligned}
I_{2} & \leq c \int_{\rho(x, x+h) \leq \varepsilon \rho(x, y)} \frac{1}{|h|^{n+q s}} \frac{\rho(x, x+h)^{\frac{q}{2}}}{\rho(x, y)^{q\left(Q-\frac{1}{2}\right)}} d h \\
& \leq \int_{\rho(x, x+h) \leq \varepsilon \rho(x, y)} \frac{c}{|h|^{n+q s}} \frac{\rho(x, x+h)^{4 q s} \rho(x, x+h)^{\frac{q}{2}-4 q s}}{\rho(x, y)^{q\left(Q-\frac{1}{2}\right)}} d h \\
& \leq c \int_{|h| \leq c \varepsilon \rho(x, y)} \frac{|h|^{2 q s}}{|h|^{n+q s}} \frac{\rho(x, y)^{\frac{q}{2}-4 q s}}{\rho(x, y)^{q\left(Q-\frac{1}{2}\right)}} d h \\
& =c \int_{|h|<c \rho(x, y)} \frac{\left.d h\right|^{n-q s}}{\rho(x, y)^{(Q-1+4 s) q}} \\
& =\frac{c}{\rho(x, y)^{q(Q-(1-3 s))}} \\
& \leq \frac{c}{\rho(x, y)^{q(Q-(1-\bar{s}))}}
\end{aligned}
$$

because $3 s<\bar{s}$.

Thus, the estimates of $I_{1}$ and $I_{2}$, together with (39), give

$$
\left(\int_{\Omega \times \Omega} \frac{d x d z}{|x-z|^{n+q s}}|f(x)-f(z)|^{q}\right)^{\frac{1}{q}} \leq c\left\|T_{1-\bar{s}}(|X f|)\right\|_{q} \leq c\|X f\|_{p}
$$

and the proof is finished

Acknowledgments. Authors would like to thank Ermanno Lanconelli for his encouragement and Giovanna Citti for some discussions about the freezing method.

\section{References}

[1] Biroli, M. and U. Mosco: Sobolev inequalities on homogeneus spaces. Potential Anal. 4 (1995), 311 - 324.

[2] Citti, G.: $C^{\infty}$ regularity of solutions of the Levi equation. Ann. Inst. H. Poincaré, Anal. non Lin. 15 (1998), 517 - 534.

[3] Citti, G., Lanconelli, E. and A. Montanari: On the interior $C^{\infty}$-solvability of the Dirichlet problem for the prescribed Levi-curvature equation. Atti Accad. Naz. Lincei Cl. Sci. Fis. Mat. Natur. Rend. Lincei (9) Mat. Appl. 10 (1999), $61-68$.

[4] Citti, G., Lanconelli, E. and A. Montanari: Smoothness of Lipschitz continuous graphs with non vanishing Levi curvature. Acta Math. (to appear). 
[5] Evans, L. C. and R. F. Gariepy: Measure Theory and Fine Properties of Functions. New York: CRC Press 1992.

[6] Fefferman, C. and D. H. Phong: Subelliptic eigenvalue problems. In: Conference on Harmonic Analysis in honor of Antoni Zygmund (Wadsworth Math. Ser.; eds.: W. Beckman et al.). Belmont (California, USA): Wadsworth 1983, pp. $590-606$.

[7] Folland, G. B.: Subelliptic estimates and function spaces on nilpotent Lie gropus. Ark. Mat. 13 (1975), $161-207$.

[8] Franchi, B.: Weighted Sobolev-Poincaré inequalities and ointwise estimates for a class of degenerate elliptic equations. Trans. Am. Math. Soc. 327 (1991), $125-158$.

[9] Franchi, B., Gallot, S. and R. L. Wheeden: Sobolev and isoperimetric inequalities for degenerate metrics. Math. Ann. 300 (1994), 557 - 571.

[10] Franchi, B. and E. Lanconelli: Hölder regularity theorem for a class of linear nonuniformly elliptic operators with measurable coefficients. Ann. Scuola Norm. Sup. Pisa Cl. Sci. (4) 10 (1983), 523 - 541.

[11] Franchi, B., Lu, G. and R. L. Wheeden: A relationship between Poincaré type inequalities and representation formulas in spaces of homogeneous type. Int. Math. Res. Not. (1996), No. 1, $1-14$.

[12] Franchi, B., Serapioni, R. and F. Serra Cassano: Approximation and embedding theorems for weighted Sobolev spaces associated with Lipschitz continuous vector fields. Boll. Un. Mat. Ital. B (7) 11 (1997), 83 - 117.

[13] Garofalo, N. and D. M. Nhieu: Isoperimetric and Sobolev inequalities for CarnotCarathéodory spaces and the existence of minimal surfaces. Comm. Pure Appl. Math. 49 (1996), 1081 - 1144.

[14] Garofalo, N. and D. M. Nhieu: Lipschitz continuity, global smooth approximations and extension theorems for Sobolev functions in Carnot-Carathéodory spaces. J. Anal. Math. 74 (1998), 67 - 97.

[15] Gatto, A. E. and S. Vági: Fractionals integrals on spaces of homogeneous type. In: Analysis and Partial Differential Equations (ed.: C. Sadosky). New York: Dekker 1990, pp. $171-216$.

[16] Gatto, A. E. and S. Vági: On the exponential integrability of fractional integrals on spaces of homogeneous type. Colloq. Math. 64 (1993), $121-127$.

[17] Hajłasz, P. and P. Koskela: Sobolev met Poincaré. Mem. Amer. Math. Soc. 688 (2000), 1 - 101.

[18] Hörmander, L.: Hypoelliptic second order differential equations. Acta Math. 119 (1967), 147 - 171.

[19] Jerison, D.: The Poincaré inequality for vector fields satisfying Hörmander's condition. Duke Math J. 53 (1986), 503 - 523.

[20] Lanconelli, E. and A. Montanari: An embedding theorem for non smooth vector fields of step two. Le Matematiche 54 (1999) - Supplemento pp. 111 - 124.

[21] Lanconelli, E. and D. Morbidelli: On the Poincaré inequality for vector fields. Ark. Mat. 38 (2000), $327-342$. 
[22] Maheux, P. and L. Saloff-Coste: Analyse sur le boules d'un opérateur souselliptique. Math. Ann. 303 (1995), 713 - 746.

[23] Rothschild, L. and E. M. Stein: Hypoelliptic differential operators and nilpotents groups. Acta Math. 137 (1976), 247 - 320.

Received 26.03.2001 